\title{
DISTRIBUTION AND MORPHOLOGY OF MECHANORECEPTORS IN SHOULDER AND ELBOW JOINTS CAPSULE IN THE GAZELLES (GAZELLA SUBGUTTUROSA)
}

\author{
B. Yilmaz ${ }^{1}$, R. Yilmaz ${ }^{2}$, I. S. Harem ${ }^{3}$ and I. Demircioglu ${ }^{1}$ \\ ${ }^{1}$ Department of Anatomy, Faculty of Veterinary Medicine, Harran University, Sanliurfa, Turkey. \\ ${ }^{2}$ Department of Pathology, Faculty of Veterinary Medicine, Harran University, Sanliurfa, Turkey. \\ ${ }^{3}$ Department of Histology and Embryology, Faculty of Veterinary Medicine, Harran University, Sanliurfa, Turkey. \\ Corresponding author's email: bestami2344@gmail.com
}

\begin{abstract}
This study aimed to detect nerves taking part innervation in gazelles' (Gazella subgutturosa) shoulder and elbow joint capsule and to classify mechanoreceptor responsible for this innervation via histological and immunohistochemical methods. In order to detect quantity of the neural elements 12 adult gazelle cadavers (6 females and 6 males) were used. Capsules taken from shoulder and elbow joint were fixed by formaldehyde. Histological structures of mechanoreceptors were detected with haematoxylin-eosin (H\&E) staining. Low-affinity neurotrophic receptor $\mathrm{p} 75$, protein gene product 9.5 (PGP-9.5) and protein S-100 were classified via immunohistochemical staining method. The nerve endings of positive staining were counted by light microscope at 400x magnification. Histological examination revealed four types of nerve endings in the joint capsule. Free nerve endings, Ruffini, Golgi-like and Pacini nerving ends were detected in descending order. The more mechanoreceptors were detected in elbow joint than shoulder joint. It was concluded that the mechanoreceptors taking part in proprioceptive function of joints were highly observed in shoulder and elbow joint capsule. This strong innervation was thought as a result of gazelles' fast and nimbly movement ability.
\end{abstract}

Key words: elbow joint, gazelle, joint capsule, mechanoreceptor, shoulder joint

\section{INTRODUCTION}

Mechanoreceptors are the neural elements, which work with touch and location sense and locate in soft tissue of joints (Zimmy, 1988; Del Valle et al., 1998). Four different types of joint receptors are detected (Polacek, 1961; Zimmy, 1988; Evans, 1993). Type I nerve endings, also known as Ruffini nerve endings, work as tensile receptors and there are responsible for adjusting joint position. Type I receptors show a slow adaptation character. Type II nerve endings (Pacini corpuscles) are located on skin, periost, around of joint, surface of nerve and fascia and responsible for a rapid adaptation to acceleration and deceleration reactions. Both types of nerve endings have a low threshold (Leonhardt, 1991; Hagert, 2008). Type III nerve endings are also named Golgi-like nerve endings, are similar to Pacini receptors and Ruffini nerve endings in respect of action and location, respectively. Except for these nerve endings, Type IV free nerve endings work as pain receptors and they can be found almost all tissues in body. Apart from Ruffini, Pacini, Golgi-like and free nerve endings, other nerve endings are accepted as unclassified corpuscles (Hagert, 2008). These receptors have been examined with special histological (Koch et al., 1995; Gómez-Barrena et al., 1999) or immunohistochemical staining methods
(Leonhardt, 1991). Afferent nerve endings can be truly detected by using glial cell protein S-100, low-affinity neurotrophin receptor $\mathrm{p} 75$ and protein gene product 9.5 (PGP 9.5) markers in immunohistochemical staining (Rein et al., 2013; 2014).

Gazelles (G. subgutturosa) are a type of Asian antelope and they mostly live in Middle East, Middle Asia, Iran, Afghanistan, Turkey and Caucasus (Kingswood and Blank, 1996; Çobanoğlu, 2010). They are tamed and hunted for a long time because of their economic, aesthetic and cultural values (Çobanoğlu, 2010). A lot of studies have been performed on different species of gazelles in respect of anatomic (Frey et al., 2008), behavioural (Blank and Yang, 2015), genetics (Lei et al., 2003; Bozkaya and Gürler, 2018), and clinical disciplines (Sempéré et al., 2001); however, anatomical characteristics of the joints in gazelles has not been studied yet.

This study aimed to detect nerves ending which innervate shoulder and elbow joint capsules in gazelles ( $G$. subgutturosa) and to investigate the types of mechanoreceptors through immunohistochemical markers. Thus, similarities and differences were shown in aspect of innervation of shoulder and elbow joints between gazelles and other species, in order to present a main data source for neurological treatments and further scientific studies about the joints. 


\section{MATERIALS AND METHODS}

Sample Materials: This study was carried out with the approval of the Local Ethics Committee of Animal Experiments at University of Harran, Sanliurfa (Permit Number: 006-10). Twelve adult (6 male and 6 female) gazelles were used for the study. The animals died in animal hospital and submitted to Department of Pathology for necropsy. All animals were free of neurological diseases and forelimb problems. Shoulder and elbow joint capsules samples were taken from the animals and fixed in $10 \%$ buffered formaldehyde solution until histological and immunohistochemical examinations.

Histological Examinations: The fixed tissue samples were routinely checked, and paraffin-embedded blocks were formed. Each blocked tissue was cut at a thickness of
$4 \mu$ using a microtome and transferred to normal microscope slides for histological examination. Histological sections were examined under light microscope with $\mathrm{x} 400$ magnification after H\&E staining. Nerve endings at four randomly chosen microscope area were counted.

Immunohistochemical Examinations: The paraffin blocks (cut) to a thickness of $4 \mu$ were transferred to microscope slides covered with poly- 1-lysine. Detection and distribution of $\mathrm{p} 75$, PGP 9.5 and protein S-100 in the joints were examined with Streptavidin-Biotin-Peroxidase methods. The p75, PGP 9.5 and S-100 antibodies positive staining of the nerve endings were determined as in the histological evaluation. Different antibodies and differences at staining process are illustrated at Table 1.

Table 1. Used antibodies and staining process differences.

\begin{tabular}{lcccc}
\hline \multicolumn{1}{c}{ Antibodies } & Dilution rate & Antigen retrieval & $\begin{array}{c}\text { Primer antibody } \\
\text { application heat and } \\
\text { time }\end{array}$ & Chromogen \\
\hline Mouse polyclonal anti-S100 & $1: 200$ & Citrat buffer & $37^{\circ} \mathrm{C}, 1$ hour & $\mathrm{AEC}$ \\
Mouse monoclonal anti-p75 & $1: 100$ & Citrat buffer & $37^{\circ} \mathrm{C}, 1$ night & $\mathrm{DAB}$ \\
Rabbit polyclonal anti- PGP 9.5 & $1: 300$ & Trypsin & $37^{\circ} \mathrm{C}, 1$ hour & $\mathrm{AEC}$ \\
\hline
\end{tabular}

Statistical Analyses: Classification of the mechanoreceptors in the joints was performed as Ruffini (Type I), Pacini (Type II), Golgi (Type III) and free nerve endings (Type IV) according to Freeman and Wyke (1967). For statistical analysis, averages of the numbers of nerve endings observed at four different microscope areas were used. Differences in the numbers of nerve endings in joint capsules between sexes (female or male) or joints (shoulder or elbow) were analysed by two-way analysis of variance with repeated measures. Presence of any correlation between shoulder and elbow joints in the numbers of nerve endings were estimated by using spearman correlation coefficient. For the statistical analysis SPSS 17.0 was used.

\section{RESULTS}

Histological Findings: Free nerve endings were observed under shoulder and elbow joint capsules in fibrous tissue with H\&E staining. These nerve endings were mostly located around vessels (Fig. 1A). Golgi-like (Fig. 1B) and small Ruffini nerve endings were less and rarely distributed in fibrous tissue.

Pacini corpuscles were also located in fibrous capsule and observed near blood vessels (Fig. 2A). However, the bigger Ruffini nerve endings were mostly located on near to muscles (Fig. 2B). As a result of semiquantitatively counting, the quantity of nerve endings was ranged from more to less as free nerve endings, Ruffini, Golgi-like and Pacini nerve endings; and, the more nerve endings were detected in shoulder than elbow joint.

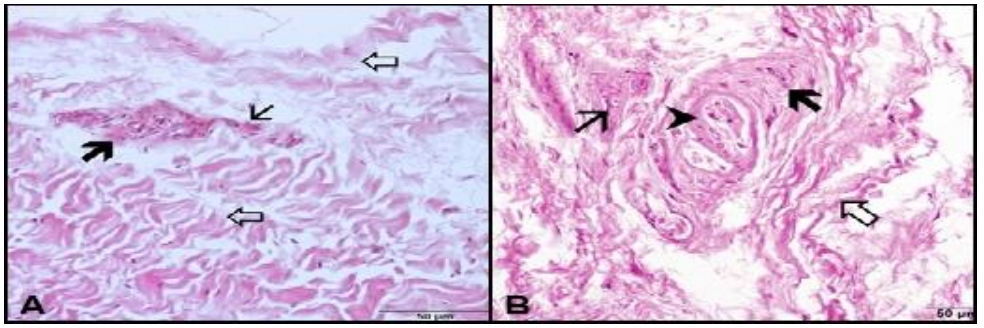

Figure 1. Light microscopic images showing of the mechanoreceptors in the shoulder joints. A. Free nerve endings (thick black arrow), blood vessel (thin arrow), fibrous tissue (thick white arrow). B. Golgi-like nerve ending (thick black arrow), Ruffini nerve ending (arrow head), blood vessel (thin arrow), fibrous tissue (thick white arrow). H\&E, X40. 


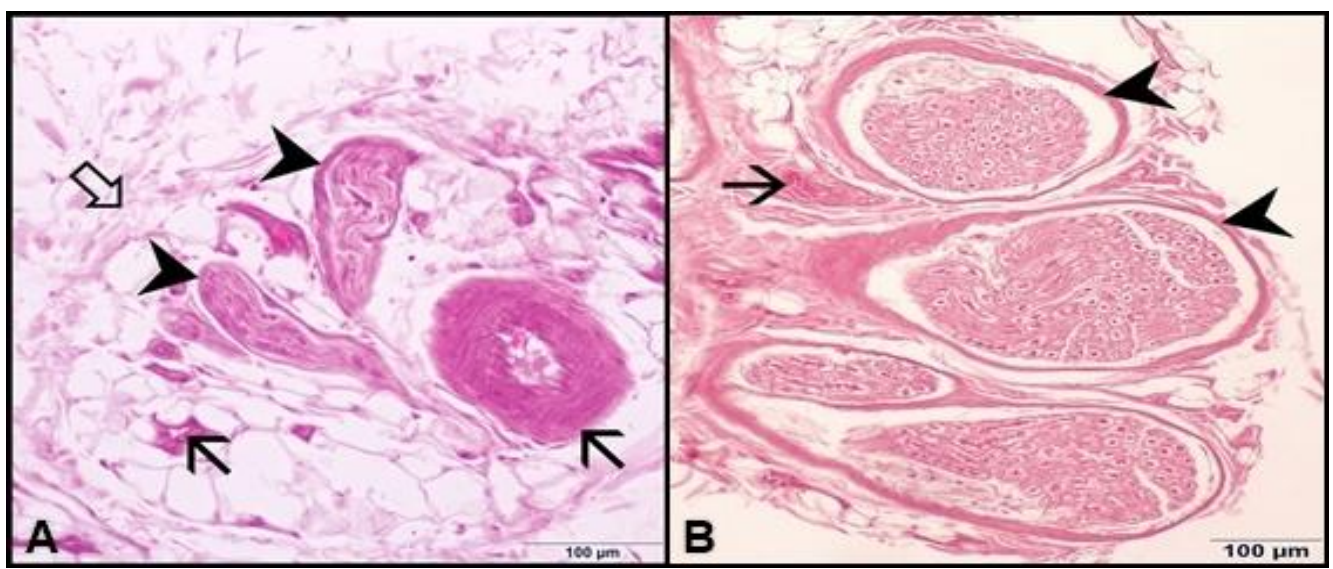

Figure 2. Light microscopic images showing of the mechanoreceptors in the elbow joints. A. Pacini nerve ending (arrow head), blood vessel (thin arrow), fibrous tissue (thick white arrow). B. Ruffini nerve ending (arrow head), blood vessel (thin arrow). H\&E, X20.

Immunohistochemical Findings: S-100: Positively stained cells were observed in nerve cell's cytoplasm as red colour at Ruffini (Fig. 3A), Pacini (Fig. 3B), Golgi- liked (Fig. 3C) and free nerve endings (Fig. 3D); and, the cell walls were observed in dark brown colour.

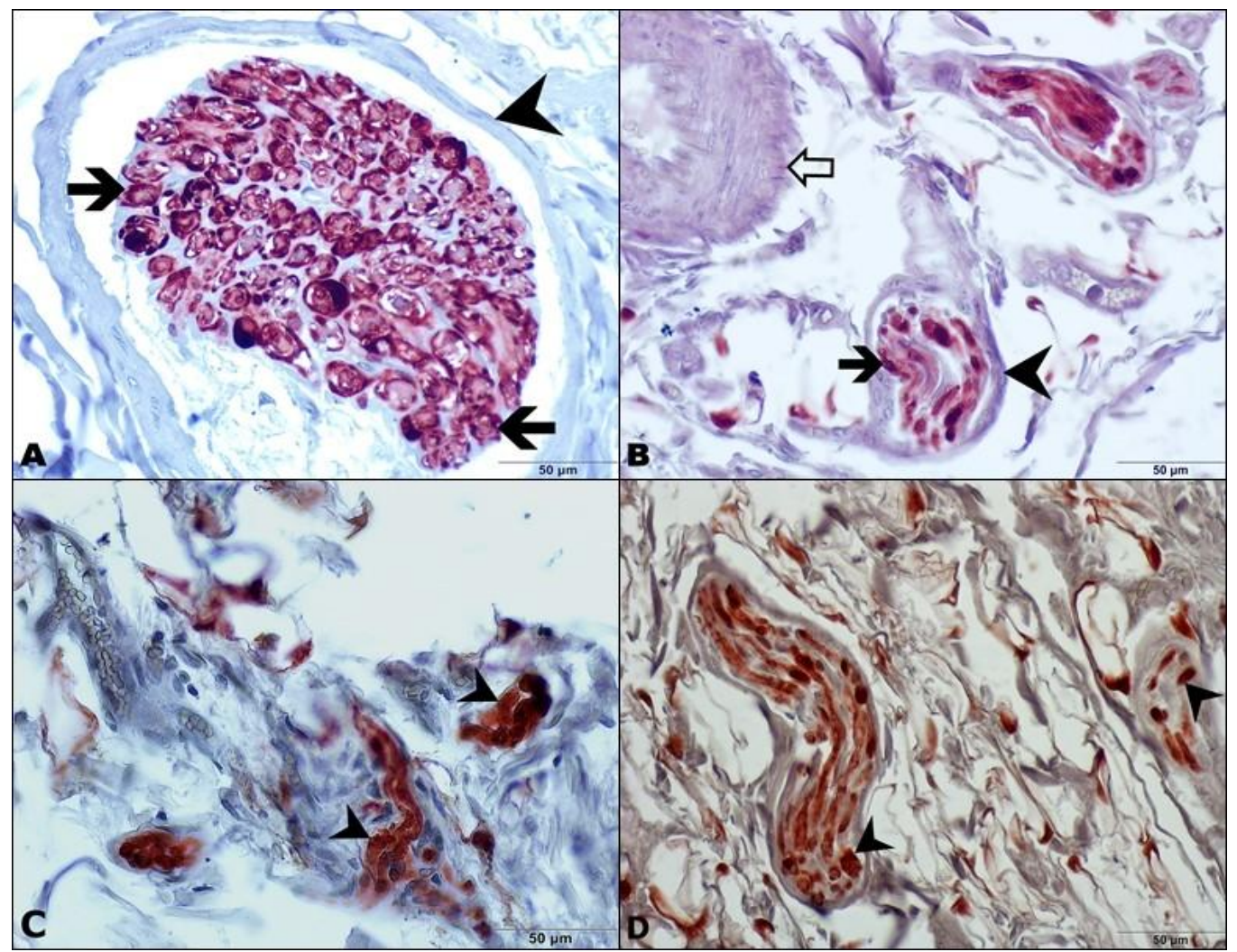

Figure 3: Immunoreactive nerve bundles for $\mathrm{S}-100$ antibody in the elbow $(A, B, D)$ and shoulder joints (C). A: Ruffini nerve ending (arrowhead), S-100 positive cells (thick black arrow). B: Pacini nerve ending (arrow head), S-100 positive cells (thick black arrow), blood vessel (white arrow). C: Golgi-like nerve endings, S100 positive cells (arrow head). D: Free nerve endings, S-100 positive cells (arrow head), IHC, X40.

PGP-9.5: Positively stained cells were detected in nerve cell's cytoplasm as red colour at Ruffini (Fig. 4A), Pacini (Fig. 4B), Golgi-liked (Fig. 4C) and free nerve endings (Fig. 4D); however, the number of the positively stained cells were less compared with antibody S-100. 


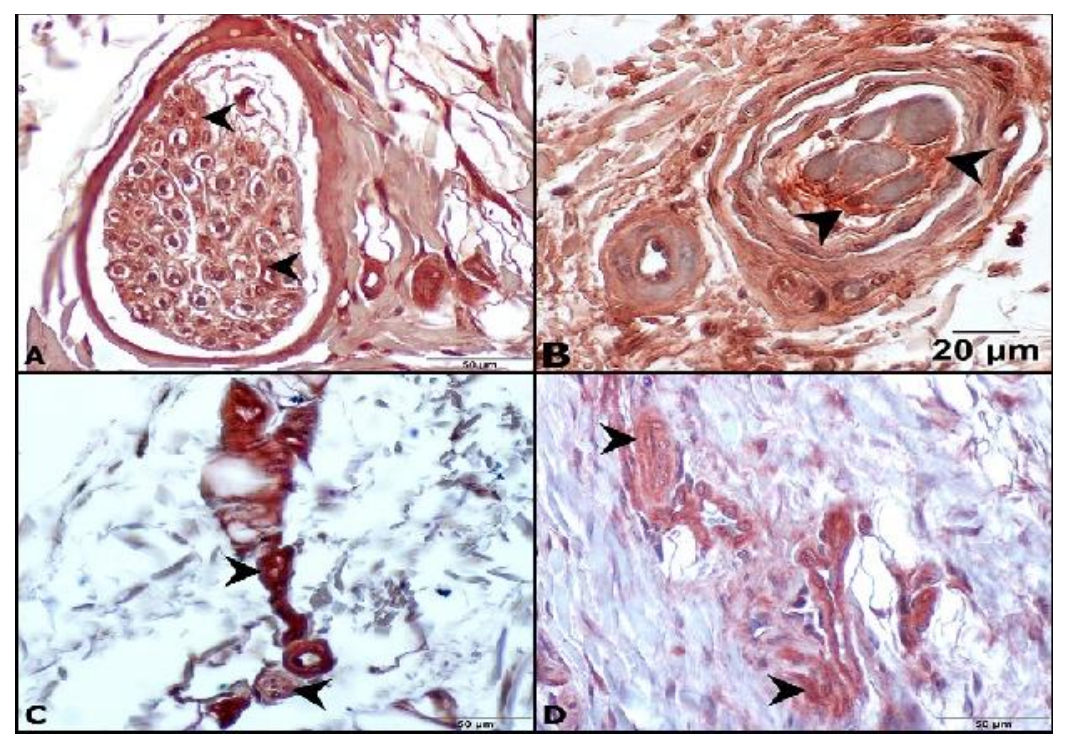

Figure 4: Immunoreactive nerve bundles for PGP-9.5 antibody in the elbow (A, C, D) and shoulder joints (B). A: Ruffini nerve ending. B: Pacini nerve ending IHC, X60. C: Golgi-like nerve ending. D: Free nerve ending. PGP-9.5 positive cells (arrow head). IHC, X40.

p-75: Positively stained cells were clearly observed on cell membrane with light brown colour at Ruffini (Fig. 5B) nerve endings, while the staining was slightly detected at Pacini (Fig. 5B), Golgi-like (Fig. 5C), and free nerve endings (Fig. 5D). The quantities of positively stained cells were significantly lower than the S-100 and PGP-9.5 antibodies.

Statistical Findings: Significant differences between shoulder and elbow joints with respect to numbers of nerve endings stained with both S-100 and PGP-9.5 $(\mathrm{p}<0.01)$, while the difference between the genders were not significant. On the other hand, the number of nerve endings stained with p-75 differed between the sexes $(p<0.05)$, while no statistically significant difference was observed between joints. No significant correlations were found in the numbers of nerve endings between two joints. The statistical analysis is shown in Table- 2 between joints and sexes the numbers of nerve endings positive stained with different antibodies.

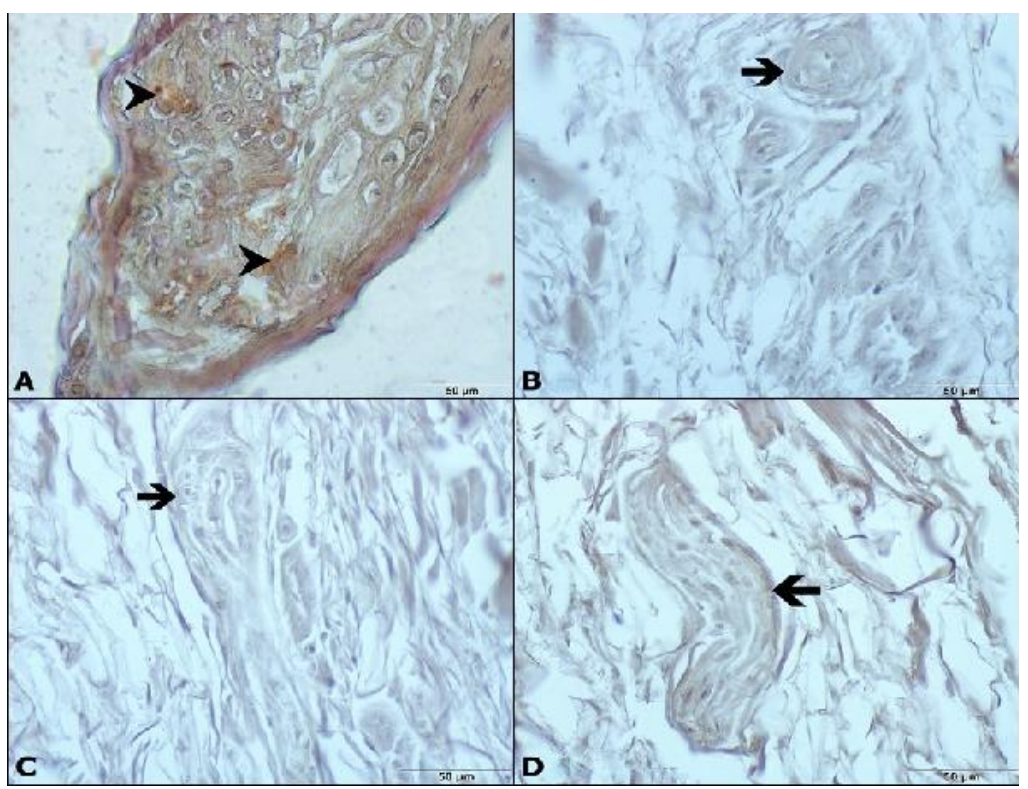

Figure 5: Immunoreactive nerve bundles for p75 antibody in the elbow joints (A, B, C, D) A: Ruffini nerve ending (arrow head) B: Pacini nerve ending (thick black arrow). C: Golgi-like nerve ending (thick black arrow). D: Free nerve ending (thick black arrow). IHC, X40. 
Table 2. Average number of nerve endings stained with different antibodies in the shoulder and elbow joint of men and female.

\begin{tabular}{lcccc}
\hline \multicolumn{5}{c}{ S 100 } \\
\hline Joint & Female & Male & All & P (Sex) \\
Shoulder & $2.333 \pm 0.12$ & $2.458 \pm 0.12$ & $2.396 \pm 0.08$ & n.s. \\
Elbow & $1.833 \pm 0.14$ & $2.042 \pm 0.14$ & $1.938 \pm 0.09$ & \\
P (Joint) & & 0.006 & & \\
\hline & PGP 9.5 & All & P (Sex) \\
\hline Joint & Female & $2.042 \pm 0.14$ & $1.938 \pm 0.09$ & n.s. \\
Shoulder & $1.833 \pm 0.14$ & $2.500 \pm 0.13$ & $2.438 \pm 0.08$ & \\
Elbow & $2.375 \pm 0.13$ & 0.006 & & P (Sex) \\
P (Joint) & & p75 & All & n.s. \\
\hline Joint & Female & $1.333 \pm 0.07$ & $1.292 \pm 0.05$ & \\
Shoulder & $1.250 \pm 0.07$ & $1.500 \pm 0.07$ & $1.354 \pm 0.06$ & \\
Elbow & $1.208 \pm 0.07$ & 0.040 & & \\
P (Joint) & & & & \\
\hline
\end{tabular}

n.s.: Not significant $(\mathrm{p}>0.05)$

\section{DISCUSSION}

Neural innervation of joint structures is controlled by peripheral receptors within tissues surrounding articular structures (Lephart, 1993). One of the key factors for successful study is the staining method. Different staining techniques and qualification methods are developed thanks to emerging technologies ( $\mathrm{Wu}$ et al., 2015). In the present study $H \& E$ and triple staining methods were preferred in order to obtain a high quality and cost-efficient display of nerve endings.

There are numerous studies performed in order to describe morphological structure and classification of nerve endings on both animals (Backenköhler et al., 1996, Schenk et al., 1996) and humans (Del Valle et al., 1998). Studies in human's nerve endings were generally focused on large joints like knee and shoulder joints (Schultz et al., 1984; Witherspoon et al., 2014). All types of nerve endings have been detected within shoulder joint capsule in both humans (Gohlke et al., 1998) and animals (Solomonow et al., 1996). Backenköhler et al. (1997) detected small lamellated Pacini, Ruffini, and Golgi-like corpuscles in shoulder joint of mouse. Free nerve endings, Ruffini and small lamellated corpuscles in joint capsules were detected by Polacek (1961). Schultz et al. (1984), did not declare exitance of Golgi tendon organs. Golgi, Pacini, Ruffini and free nerve endings were observed within shoulder and elbow joint capsules in the present study. These findings are compatible with other studies on both humans (Gohlke et al., 1998) and other mammalian species (Backenköhler et al., 1997).

It has been reported that different types of nerve endings are found at different regions of joints and also the high quantity of nerve endings are observed at end points related with movement of animal (Zimmy, 1988). Ruffini corpuscles have been detected in stratum fibrosum of adipose tissues and ventral surface of shoulder joint in small numbers while they have not been observed in synovial membrane of shoulder joint capsule in laboratory mouse (Backenköhler et al., 1996; 1997). A high quantity of Ruffini corpuscles were detected on joints capsules, in coracoacromial ligament and rotator cuff in human (Gohlke et al., 1998). Golgi tendon organs have mostly been detected in shoulder region near to joint capsules and at the muscle-tendon junctions in mouse (Wohlfart and Henriksson, 1960; Barker, 1974; Backenköhler et al., 1997). As a result of semiquantitative study on human Gohlke et al. (1998) have reported that highest number of Pacini nerve endings are found in the joint capsules of shoulder joint. Solomonow et al. (1996) found mostly free nerve endings in cat shoulder joint. Although Maass et al. (2001) have detected numerous small lamellar corpuscles around shoulder soft tissue, they reported that the number of Pacini and Ruffini corpuscles were contrarily less on gray short tailed opossum (Monodelphis domestica). In accordance with previous studies (Backenköhler et al., 1996, Rein et al., 2013; 2014) our histological findings indicated that the nerve endings located in fibrous tissue within joint capsules.

In the present study the free nerve endings were located in fibrous tissue under joint capsule near vessels suggesting a functional role associated with the stability and strength of the joint (Schultz et al., 1984) as well as with nutritional support of the joint in order to start quick recovery and fast impulse transmission in case of inflammation and injury (Zimmy, 1988). In contrast to free nerve endings the number of the Golgi-like and small Ruffini nerve endings were low in fibrous tissue. Although, Pacini nerve endings were also observed in the fibrous capsule, they were located near blood vessels. In contrast to an earlier study (Backenköhler et al., 1996) 
numerous larger Ruffini nerve endings were detected near to muscles.

Immunohistochemical examinations in the present study showed that density of the cells ranked as S100, PGP-9.5 and p-75. Additionally, the ranking of the number of nerve endings in shoulder and elbow joints were as free nerve endings, Ruffini, Golgi-like and Pacini corpuscles. Anatomical studies (Y1lmaz and Bahadir, 2009; 2011) revealed that number of proprioceptive nerve branches in elbow joint is higher than that in shoulder joint. In the present study density of nerve endings in elbow joint was found to be higher than in shoulder joint. Therefore, we suggested that the reason of the higher density in elbow joint may be the higher number of proprioceptive nerve branches innerving this joint.

Several suggestions have been declared relating the functions of the mechanoreceptors in the joints based of anatomical, histological and neurological studies performed in human and animals. Pacini, Ruffini, Golgi and free nerve endings are described as the root of the proprioceptive traits (Gómez-Barrena et al., 1999; Pötzl et al., 2004). It is believed that encapsulated nerve endings give proprioceptive and supporting information to central nervous system regarding to the joint function and position. By using a surgical method Pötzl et al. (2004) have shown that proprioceptive capacity plays an important role for articular stability and coordination on the shoulder. Kallakuri et al. (2012) have hypothesized that Pacini-like nerve endings play a vital role for the changing of joint position at high speed by innervation of the cervical facet joint capsule in human.

Conclusion: Consequently, the present study was the first neuroanatomic research for investigating type and distribution of the shoulder and elbow joints mechanoreceptors in gazelles. We suggest that the large quantity of the mechanoreceptors in and around the joint capsule is associated with not only the shoulder and elbow stability but also species-typical movement and agility of gazelles. Furthermore, the study will contribute to further functional anatomy research and provide anatomical and histological information for developing new clinical treatment protocols.

Acknowledgements: This work was supported by the Scientific Research Project Fund of Harran University under the project number 15089.

\section{REFERENCES}

Backenköhler, U., Z. Halata, and T.J. Strasmann (1996). The sensory innervation of the shoulder joint of the mouse. Ann. Anat. 178: 173-181.

Backenköhler, U., T.J. Strasmann, and Z. Halata (1997). Topography of mechanoreceptors in the shoulder joint region-a computer-aided $3 \mathrm{D}$ reconstruction in the laboratory mouse. Anat Rec. 248: 433-441.
Barker, D. (1974): The morphology of muscle receptors. In: Hunt, C.C (eds), Muscle Receptors. Handbook of Sensory Physiology. Volume III/2, Springer Verlag, Berlin.

Blank, D. and W. Yang (2015). Suckling behavior in goitered gazelle: do females invest more in twins or singletons? Zoology. 118: 348-356.

Bozkaya, F. and S. Gürler (2018). Sequence diversity of MHC class-II DRB gene in gazelles (Gazella subgutturosa) raised in Sanliurfa of Turkey. J. Genet. 97: 897-903.

Çobanoğlu, A.E. (2010). Identification of demographic structure and population viability analysis of Gazella subgutturosa in terms of sex ratio and age classes in Urfa Ceylanpinar. Msc thesis, Middle East Technical University, Ankara, Turkey.

Del Valle, M.E., S.F. Harwin, A. Maestro, A. Murcia, and J.A. Vega (1998). Immunohistochemical analysis of mechanoreceptors in the human posterior cruciate ligament: a demonstration of its proprioceptive role and clinical relevance. $\mathrm{J}$. Arthroplasty. 13: 916-922.

Evans, H.E. (1993). Arthrology. In: Evans, H.E (ed) Miller's anatomy of the dog. Third ed., W.B. Saunders Company Philadelphia, London, Toronto. pp. 219-257.

Freeman, M.A., and B. Wyke (1967). The innervation of the knee joint. An anatomical and histological study in the cat. J. Anat. 101: 505- 532.

Frey, R., A. Gebler, K.A. Olson, D. Odonkhuu, G. Fritsch, N. Batsaikhan, and I.W. Stuermer (2008). Head anatomy of male and female mongolian gazelle-a striking example of sexual dimorphism. In: Endo, H., Frey, R (eds), Anatomical Imaging. Springer, Tokyo. pp. 1-13.

Gohlke, F., E. Janssen, J. Leidel, B. Heppelmann, and J. Eulert (1998). Histomorphological findings in the proprioception of the shoulder joint. Orthopäde. 27: 510-517.

Gómez-Barrena, E., E. Martinez-Moreno, R. Ballesteros Massó, D. Martinez Pérez, and L. Munuera Martinez (1999). Gold chloride technique to study articular innervation. A protocol validated through computer-assisted colorimetry. Histol. Histopathol. 14: 69-79.

Hagert, E. (2008). Wrist ligaments innervation patterns and ligamento-muscular reflexes. $\mathrm{PhD}$ thesis, Karolinska Institutet, Stockholm, Sweden.

Kallakuri, S., Y. Li, C. Chen, and J.M. Cavanaugh (2012). Innervation of cervical ventral facet joint capsule: Histological evidence. World J Orthop. 3: 10-14.

Kingswood, S.C. and D.A. Blank (1996). Gazella subgutturosa. Mammal Sp. 518: 1-10.

Koch, B., G. Kurriger, and R.A. Brand (1995). Characterisation of the neurosensory elements of 
the feline cranial cruciate ligament. J Anat. 187: 353-359.

Lei, R., Z. Hu, Z. Jiang, and W. Yang (2003). Phylogeography and genetic diversity of the critically endangered Przewalski's gazelle. Anim Conserv. 6: 361-367.

Leonhardt, H. (1991). Histologie, zytologie und mikroanatomie des Menschen, 6th edn. Thieme Verlag, Stuttgart.

Lephart, S.M. (1993). Reestablishing proprioception, kinesthesia, joint position sense, and neuromuscular control in rehabilitation. In: Prentice, WE (ed) Rehabilitation techniques in sports medicine, 2nd edn. Times Mirror Mosby College Publishing, St. Louis, Missouri. p.145.

Maass, S., K.I. Baumann, and Z. Halata (2001). Topography of muscle spindles and golgi tendon organs in shoulder muscles of "Monodelphis domestica". Ann Anat. 183: 237-242.

Polacek, P. (1961). Differences in the structure and variability of encapsulated nerve endings in the joints of somespecies of mammals. Acta Anatomica. 47: 112-124.

Pötzl, W., L. Thorwesten, C. Götze, S. Garmann, and J. Steinbeck (2004). Proprioception of the shoulder joint after surgical repair for instability: a longterm follow-up study. Am J Sports Med. 32: 425430.

Rein, S., U. Hanisch, H. Zwipp, A. Fieguth, S. Lwowski, and E. Hagert (2013). Comparative analysis of inter- and intraligamentous distribution of sensory nerve endings in ankle ligaments: a cadaver study. Foot Ankle Int. 34: 1017-1024.

Rein, S., S. Manthey, H. Zwipp, and A. Witt (2014). Distribution of sensory nerve endings around the human sinus tarsi: a cadaver study. J Anat. 224: 499-508.

Schenk, I., A. Spaethe, Z. Halata (1996). The structure of sensory nerve endings in the knee joint capsule of the dog. Ann Anat. 178: 515-521.
Schultz, R.A., D.C. Miller, C.S. Kerr, and L. Micheli (1984). Mechanoreceptors in human cruciate ligaments: a histological study. J Bone Joint Surg Am. 66: 1072-1076.

Sempéré, A.J., N. Brown, O.B. Pereladova, K. Bahloul, A. Lacroix, and N. Soldatova (2001). Comparative analysis of reproductive cycles in female Persian gazelle (Gazella subgutturosa subgutturosa) (Central Asia) and sand gazelle (Gazella subgutturosa marica) (Arabian Peninsula). Gen Comp Endocrinol. 121: 57-65.

Solomonow, M., C. Guanche, C. Wink, T. Knat, R.V. Baratta, and Y. Lu (1996). Mechanoreceptors and reflex arc in the feline shoulder. J Shoulder Elb Surg. 5: 139-146.

Witherspoon, J., I.V. Smirnova, and T.E. McIff (2014). Neuroanatomical distribution of mechanoreceptors in the human cadaveric shoulder capsule and labrum. J Anat. 225: 337 45.

Wohlfart, G., and K.G. Henriksson (1960). Observations on the distribution, number, and innervation of Golgi musculo-tendinons organs. Acta Anat. 41: 192-204.

Wu, X., W. Song, C. Zheng, S. Zhou, and S. Bai (2015). Morphological study of mechanoreceptors in collateral ligaments of the ankle joint. J Orthop Surg Res. 10: 92.

Yilmaz, B. and A. Bahadır (2009). Nerves innervating articular capsule of elbow joints in turkish shepherd dogs (Karabaş - Kangal), curly sheep and native hair goats: an anatomic study. Kafkas Univ Vet Fak Derg. 15: 835-840.

Yilmaz, B. and A. Bahadir (2011). Innervation of the shoulder joints in turkish shepherd dogs (Karabash-Kangal), curly sheep and native hair goats: an anatomic study. J Anim Vet Adv. 10: 1934-1937.

Zimmy, M.L. (1988). Mechanoreceptors in particular tissues. Am J Anat. 182: 16-32. 